\title{
The Role of Ce Doping in Enhancing Sensing Performance of ZnO-based Gas Sensor by Adjusting the Proportion of Oxygen Species
}

Yiqun Zhang, Yueying Liu, Linsheng Zhou, Deye Liu, Fengmin Liu*, Fangmeng Liu, Xishuang Liang, Xu Yan, Yuan Gao, Geyu Lu*

State Key Laboratory of Integrated Optoelectronics, Key Laboratory of gas sensors, Jilin Province and College of Electronic Science and Engineering, Jilin University, 2699 Qianjin Street, Changchun 1300

12, People's Republic of China

(Corresponding authors:

Fengmin Liu, Tel.: +86 431 85168384; fax: +86 431 85167808;

Email: liufm@jlu.edu.cn.

Geyu Lu, Tel.: +86 431 85167808; fax: +86 431 85167808;

Email: lugy@jlu.edu.cn)

\begin{abstract}
:
Flower-liked $\mathrm{ZnO}$ architectures and Ce doped $\mathrm{ZnO}$ materials with different amounts $(0.2,0.5,1.2$ and 2 at\% Ce) were successfully synthesized by a simple room-temperature precipitation route. As the gas sensing materials, their sensing performance were investigated systematically. The results indicate that $\mathrm{Ce}$ doping can improve the performance of $\mathrm{ZnO}$ sensor. The $\mathrm{ZnO}$ doped with 0.5 at $\% \mathrm{Ce}$ exhibited the highest response to ethanol at the operating temperature of $300^{\circ} \mathrm{C}$, and the response value was about 72.6 to $100 \mathrm{ppm}$ ethanol. With Ce doping, the proportions of oxygen vacancy and chemisorbed oxygen species were increased obviously, which could greatly promote the gas sensing properties of surface resistance-type metal oxide semiconductors. Thus, the doping of flower-liked $\mathrm{ZnO}$ with $\mathrm{Ce}$ should be a promising approach for designing and fabricating the high performance gas sensor.
\end{abstract}

Key words: $\mathrm{Ce}, \mathrm{ZnO}$, oxygen species, gas sensor, ethanol

\section{Gas sensing performances}
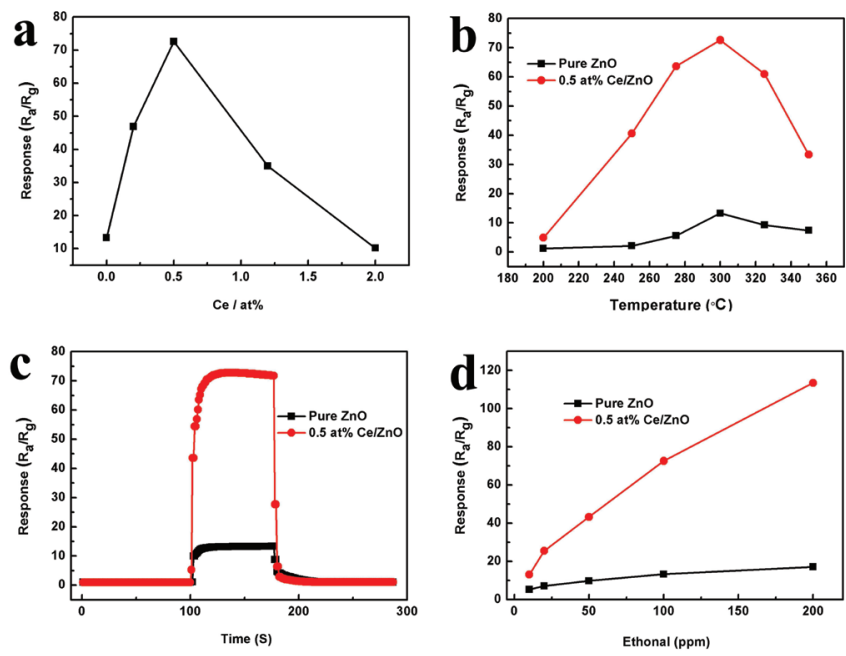
Fig. 1. Response of the sensors based on pure $\mathrm{ZnO}$ and Ce doped $\mathrm{ZnO}$ materials with different Ce doping amounts (a), responses at different operating temperatures (b) and dynamic response curves to 100 ppm ethanol (c) as well as responses to different concentrations of ethanol (d) of the sensors based on pure $\mathrm{ZnO}$ and 0.5 at\% $\mathrm{Ce} / \mathrm{ZnO}$ composites.
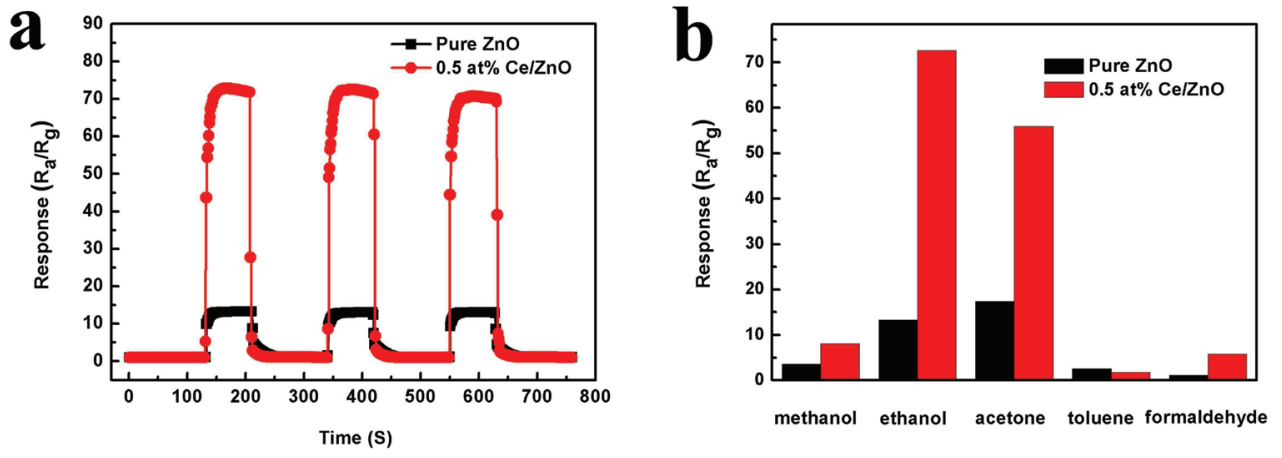

Fig. 2. Three reversible cycles of the sensors based on pure $\mathrm{ZnO}$ and 0.5 at $\% \mathrm{Ce} / \mathrm{ZnO}$ composites for successive detection of 100 ppm ethanol (a), and responses of the sensors to $100 \mathrm{ppm}$ of various gases (b).

\section{References}

[1] L. Zhu, Y.Q. Li, W. Zeng, Enhanced Ethanol Sensing and Mechanism of Cr-doped $\mathrm{ZnO}$ Nanorods: Experimental and Computational Study, Ceramics International 43, 14873-14879 (2017); doi: 10.1016/j.ceramint.2017.08.003

[2] L. Zhu, Y.Q. Li, W. Zeng, Hydrothermal Synthesis of Hierarchical Flower-like ZnO Nanostructure and its Enhanced Ethanol Gas-sensing Properties, Applied Surface Science 427, 281287 (2018); doi: 10.1016/j.apsusc.2017.08.229

[3] J.J. Guo, C.Peng, Synthesis of $\mathrm{ZnO}$ Nanoparticles with a Novel Combustion Method and their $\mathrm{C}_{2} \mathrm{H}_{5} \mathrm{OH}$ Gas Sensing Properties, Ceramics International 41, 2180-2186 (2015); doi: 10.1016/j.ceramint.2014.10.017

[4] J. Guo, J. Zhang, M. Zhu, D.X. Ju, H.Y. Xu, B.Q. Cao, High-performance Gas Sensor Based on $\mathrm{ZnO}$ Nanowires Functionalized by $\mathrm{Au}$ Nanoparticles, Sensors and Actuators B 199, 339-345 (2014); doi: 10.1016/j.snb.2014.04.010

[5] Y. Li, G.Z. Li, Y.L. Zou, Q. Wang, Q.J. Zhou, X.X. Lian, Preparation and Sensing Performance of Petal-like $\mathrm{RuO}_{2}$ Modified $\mathrm{ZnO}$ Nanosheets via a Facile Solvothermal and Calcination Method, Trans, Nonferrous Met. Soc. China 24, 28962903 (2014); doi: 10.1016/S10036326(14)63424-5

[6] T. Tharsika, A. S. M. A. Haseeb, Sheikh A. Akbar, Mohd Faizul Mohd Sabri and Wong Yew Hoong, Enhanced Ethanol Gas Sensing Properties of $\mathrm{SnO}_{2}$-Core/ ZnO-Shell Nanostructures, Sensors 14, 14586-14600 (2014); doi: $10.3390 / \mathrm{s} 140814586$ 